\title{
Arrhythmia Detection Using Deep Belief Network Extracted Features From ECG Signals
}

\author{
Mahendra Kumar Gourisaria, KIIT University (Deemed), India \\ (iD) https://orcid.org/0000-0002-1785-8586 \\ Harshvardhan GM, KIIT University (Deemed), India \\ Rakshit Agrawal, KIIT University (Deemed), India \\ Sudhansu Shekhar Patra, KIIT University (Deemed), India \\ iD https://orcid.org/0000-0001-9996-7681 \\ Siddharth Swarup Rautaray, KIIT University (Deemed), India \\ Manjusha Pandey, KIIT University (Deemed), India
}

\begin{abstract}
Arrhythmia is a disorder of the heart caused by the erratic nature of heartbeats occurring due to conduction failures of the electrical signals in the cardiac muscle. In recent years, research has been done towards accurate categorization of heartbeats and electrocardiogram (ECG)-based heartbeat processing. Accurate categorization of different heartbeats is an important step for diagnosis of arrhythmia. This paper primarily focuses on effective feature extraction of the ECG signals for model performance enhancement using an unsupervised deep belief network (DBN) pipelined onto a simple logistic regression (LR) classifier. The authors compare and evaluate the results of data feature enrichment against plain, non-enriched data based on the metrics of precision, recall, specificity, and F1-score and report the extent of increase in performance. Also, they compare the performance of the DBN-LR pipeline with a 1D convolution technique and find that the DBN-LR algorithm achieves a 5\% and $10 \%$ increase in accuracy when compared to $1 \mathrm{D}$ convolution and no feature extraction using DBN, respectively.
\end{abstract}

\section{KEYWORDS}

Boltzmann Machines, Deep Belief Networks, Deep Neural Networks, ECG-Based Heartbeat Detection, Feature Extraction, Logistic Regression, Machine Learning

\section{INTRODUCTION}

Heart arrhythmia is a category of heart disease which results from any disturbances in the rate, uniformity, and site origin or conduction of the cardiac electric impulse (Thaler, 1999). By examining and analyzing the combination of action impulse waveforms of the electrical signal of each heartbeat which are produced by various specialized cardiac tissues present in the heart, detection

DOI: 10.4018/IJEHMC.20211101.oa9

This article published as an Open Access article distributed under the terms of the Creative Commons Attribution License (http://creativecommons.org/licenses/by/4.0/) which permits unrestricted use, distribution, and production in any medium, provided the author of the original work and original publication source are properly credited. 
Table 1. Categorization of heartbeats using AAMI EC57 (Association for the Advancement of Medical Instrumentation, 1998) categories and annotations

\begin{tabular}{|l|l|}
\hline \multicolumn{1}{|c|}{ Annotations } & Category \\
\hline Normal beats / Left or Right bundle branch block & N \\
\hline $\begin{array}{l}\text { Supraventricular ectopic beats / Aberrant atrial premature / } \\
\text { Supra-ventricular premature / Nodal premature }\end{array}$ & S \\
\hline Ventricular ectopic beats / Ventricular Escape & V \\
\hline Fusion beats (of ventricular and normal) & F \\
\hline $\begin{array}{l}\text { Unknown or Unclassifiable beats (Paced or fusion of paced and } \\
\text { normal) }\end{array}$ & Q \\
\hline
\end{tabular}

of abnormalities is possible (Luz et al., 2016). For the diagnosis of cardiac arrhythmia or any other heart rhythm disorder, electrocardiogram (ECG) is one of the most frequently used methods which helps in measuring and monitoring the impulses of the heart in the form of waveforms as it is noninvasive and efficient as well as an accurate method that provides useful information related to the heart impulse and other required details.

The muscles of the human heart contract and relax rhythmically for blood circulation throughout the body. Atrial sine node being the site of initial contractions act as a natural pacemaker and further pass through the other muscles. These electric signals propagate in a specific manner and follow a pattern (Herone and Smith, 2003), generating light electrical currents on the surface of the skin. These electrical signals are then measured with the help of electrodes and other appropriate devices. Electrodes measure the electric potential difference between the points and are amplified with optic isolation using suitable devices. Then these generated signals are passed through a high pass, followed by a low pass filter. At last, these signals are finally converted from analog signals to digital signals. This process of generating a graphical representation of digital signals done by the electrocardiogram (ECG) (Luz et al., 2016).

Recently, heart diseases have emerged as a major problem due to high rates of incidence and mortality. The treatment cost is another factor making it a serious disease as it requires long-term treatment and frequently entails expensive therapies for cure (Mozzafarian et al., 2015; National Center for Health Statistics, 2005). Every year, 17.34 million deaths are recorded owing to diseases that are related to the heart which in total accounts for $37 \%$ of the total deaths globally (Smith et al., 2005; Healthsquare, 2007; World Health Organization, 2014). Due to these reasons, with an expected progressive aging of the population globally, it may lead to an increased number of deaths from 17 million in 2016 to 24 million by 2030 (de Chazal et al., 2004). Many heart-related diseases can be diagnosed early via machine learning techniques (Anand et al., 2020; Das et al., 2020) and frequent itemset mining (Nayak et al., 2019). Several cases of arrhythmia appear as sequences of heartbeats with different timing or variation of ECG morphology. Classification of the correct type of heartbeat is an important step for the diagnosis of arrhythmia. The electric rhythm generated from the ECG signal is determined by analyzing and determining the consecutive heartbeats in the signal (Elhaj et al., 2016). According to the Association for the Advancement of Medical Instrumentation (AAMI), a heartbeat is further classified into five different categories which help in the diagnosis of arrhythmia (Association for the Advancement of Medical Instrumentation, 1998; Spach and Kootsey, 1983). Table 1 shows the categories of irregular heartbeats with their respective annotations.

According to the American Heart Association (AMA), over 70 million people around the world had been diagnosed with cardiovascular diseases (Elhaj et al., 2016). Data on the country-wise spread of arrhythmia or atrial fibrillation remains largely unorganized and inaccessible in general media. This may be due to poor surveillance across various developing and even developing countries. 
Moreover, (Sidney et al., 2013) the amassment of the data provided by the Heart Disease and Stroke Statistics Update does not comprise timely and representative data on stroke incidence and heart diseases in the USA.

The problem statement that we tackle through this paper is to correctly classify the category of arrhythmia (N, S, V, F, or Q) given the digitized ambulatory ECG recordings of a mixed cohort of patients recorded at 360 samples per second per channel (Mark and Moody, 1998). Techniques such as $1 \mathrm{D}$ convolution ( $\mathrm{Li}$, D. et al., 2017) require complex preprocessing of the signals before the data is deemed suitable for the 1D convolution. This necessitates the use of prominent single-step feature extraction practices working over low-level preprocessed data so that the model formation complexity is reduced with little-to-no compromises made on detection metrics.

Related technology mainly used for the detection of the category of arrhythmia include 1D convolution, adaptive filtering before detection (Thakor and Zhu, 1991), use of non-linear dynamical modeling (Owis et al., 2002), supervised neural networks (Hannun et al., 2019), autocorrelation function (Guillén et al., 1990), via time-frequency analysis (Afonoso and Tompkins, 1995), using wavelet transform (Khadra et al., 1997), etc. Adaptive filtering makes use of various structures of filters to get rid of noise, these structures are baseline wander, $60 \mathrm{~Hz}$ power line interference, motion effect, and muscle noise. In this context, adaptive recurrent filter structures may be used (Thakor and Zhu, 1991) to analyze ECG signals and to categorize them. Another traditional method to model arrhythmia detection is by using non-linear dynamical modeling (Owis et al., 2002) where five categories of ECG are classified using the largest Lyapunov exponent and correlation dimension. Apart from the traditional approaches, because of recent developments made in machine and deep learning, supervised neural networks have been employed for the detection of arrhythmia as done in (Hannun et al., 2019). However, in this paper, we employ the unsupervised counterparts of traditional artificial neural networks that belong to the Boltzmann machine family (GM et al., 2021a). We use a generative model (GM et al., 2020) to generate high level features extracted from the ECG signals that resemble the original data distribution.

All the samples that are obtained from the MIT-BIH database have a constant frequency of $125 \mathrm{~Hz}$. Process of arrhythmia identification and classification can be difficult for humans to detect because at times it becomes a mandatory job to monitor each heartbeat from the ECG records which might require sitting of hours and hours or even days and hence, there are high chances of encountering a human error during the process of analyzing due to ambiguity in the signal forms. Today, machine learning techniques have eased the burden of pattern recognition from humans in almost every sector of the industry. From finance such as in credit card fraud detection (Sahu et al., 2020) to web analytics in sentiment analysis (Chandra et al., 2021), machine learning has made a profound impact. However, even for machine learning models, such ambiguities make performance inconsistent over classifying the types of new a patient's signals. To avoid this, in this paper, we present a powerful feature extraction tool using a Bayesian network namely Deep Belief Network (DBN) which falls in the part of unsupervised deep learning. Classifier applied on the feature extracted vector done by the DBNs shows superior performance as opposed to no feature extraction and helps in automatic decision making and categorizing the type of arrhythmia disease very accurately. Our contributions in this paper are as follows:

1. We use an unconventional method of unsupervised deep learning namely deep belief network to extract useful ECG features.

2. A logistic regression classifier is used to work upon the DBN features to form an end-to-end pipeline for the categorization of arrhythmias.

3. Various metrics such as precision, recall, specificity, and F1-score are used for comparison of results.

4. We implement another approach that makes use of $1 \mathrm{D}$ convolution for comparison with the DBN approach. 
5. We present a comparison of the results of work with other studies done in the past.

The main novelty of our work lies in the fact that, to the best of our knowledge, no study has used DBN in the past and done a comprehensive analysis of the results by i) comparison with an unconventional technique such as 1D convolution, ii) with more than three performance metrics, and iii) by providing a comparison as to how no extraction of features using DBN affects performance (as we shall see in Section 4).

The rest of the paper is organized as follows: 2. Related work, 3. Methodology and Data, 4. Experimentation and results, 5. Future Directions and 6. Conclusion. An analytical and comprehensive evaluation of the applied algorithm has been done and also been compared with other work done in the literature.

\section{RELATED WORK}

Many researchers are in the process of creating new algorithms and techniques for the development and advancement of medical diagnostic tools. Lately, different techniques using neural networks have been implemented for the diagnosis as well treatment of various diseases with overwhelming results. Different researches have mainly focused on feature extraction and selection of a small dataset of non-redundant, predictive features for ECG graphical representation. Authors in (Ince et al., 2009; Jiang and Kong, 2007; Martis et al., 2013; Ye et al., 2012) have implemented different types of feature extraction like Hermite transform, discrete wavelet transform, and independent component analysis. Different types of feature extraction were combined and used sequentially to optimize the set of discriminant features for the classification of arrhythmia type (Chen et al., 2017; Mar et al., 2011; Teijeiro et al., 2018). Authors in (Mar et al., 2011) employed used an algorithm named as sequential forward floating search to select prominent features over which a multilayer perceptron is trained. In (Chen et al., 2017), an ECG beat classification is performed based on a mixture of dynamic and projected features. Various classifiers based on the ECG features have been implemented in the study which includes hidden Markov models (Pan et al., 2012), MOE (mixture of experts) approach (Hu et al., 1997), SVM (support vector machine) (Chen et al., 2017), BBNN (blocked-based neural network) (Jiang and Kong, 2017), regression neural networks (Li, P. et al., 2017), genetic algorithmback propagation neural network ( $\mathrm{Li}, \mathrm{H}$. et al., 2017), and artificial neural networks (Jadhav et al., 2010; Sannino and Pietro, 2018).

In recent studies, it is found that convolutional neural networks (CNNs)-based models with feature enrichment show accurate and efficient results in analyzing not only ECG signals but also EEG (Electroencephalogram) signals (Acharya et al., 2017b, 2018). LeCun et al. (1990) implemented CNN back in 1990 which today is considered to be one of the flagship neural networks. Rapid development in GPU (Graphics Process Unit) technology helps CNN in outperforming with much higher accuracy and efficiency with much lesser loss in image recognition (Krizhevsky et al., 2012), audio classification (Hinton et al., 2012), and many more. CNN shows promising applications in time series bio-signals like ECG (Acharya et al., 2017a; Al Rahhal et al., 2016; Kiranyaz and Gabbouj, 2016; Yildırım et al., 2018).

In the study proposed by $\mathrm{Hu}$ et al. (1997), work is done on customization of different heartbeat classifiers of specific patients and then combined with the classifiers that were developed from the ECG signal database. The MOE approach was used in the study to combine the classifiers. The paper reported $62.2 \%$ of accuracy while $94 \%$ accuracy with MOE classifier when categorizing ventricular ectopic beats from non-ventricular ectopic beats. Another study by Lagerholm et al. (2000) mentioned a method involving the formation of clusters or groups of $25 \mathrm{ECG}$ records which gave an experimental accuracy of $98.5 \%$ of the correctness of the prediction of the group of the heartbeat signals. Rajpurkar et al. (2017) worked on the classification of atrial fibrillation and implemented it on 30000 patients' ECG signals. 
Different approaches of CNN are used in classifying the ECG signals. For instance, Inan et al. (2006) used dyadic wavelet transform and neural networks to achieve $95.16 \%$ accuracy. Sayadi et al. (2010) used the Kalman and Bayesian filters to get an accuracy of $99.10 \%$, with $98.77 \%$ of sensitivity and $97.4 \%$ in specificity. Martis et al. (2011) implemented higher-order statistics, wavelet packet decomposition, and SVM (support vector machine) classifier to obtain accuracy, sensitivity, and specificity as $98.40 \%, 98.90 \%$, and $98 \%$ respectively, whereas, Prasad et al. (2013) used three approaches: higher-order statistics, independent component analysis, and KNN (K-nearest neighbors) classifier for the model and reported results as $97.65 \%$ of accuracy, $98.75 \%$ of sensitivity, and $99.53 \%$ of specificity. Li et al. (2016) used wavelet packet decomposition and RR intervals with wavelet packet entropy and random forest classifier to predict the targets with an accuracy of $94.61 \%$. Another study proposed by Acharya et al. (2017b) implemented a nine-layer CNN, who divided the whole dataset into two parts: with noise and without noise and worked in the generation of synthetic data and achieved the results in accuracy, sensitivity, and specificity of set 1: 93.47\%, 96.01\%, and 91.64\% respectively and for set 2: $94.03 \%, 96.71 \%$, and $91.54 \%$.

In this paper, we use feature enrichment using DBNs of the preprocessed (the preprocessing details are given later in Section 3.2) ECG signals before training a logistic classifier model to improve the overall accuracy and minimize the losses. We also compare this approach to a 1D convolutional neural network performing ECG detections on the same dataset.

\section{METHODOLOGY AND DATA}

This section contains the methods used in our feature extraction-based ECG signal classification model. We also specify the preprocessing of raw ECG data to transform data into a suitable form for the DBN. The section is further divided into subsections: 3.1 Dataset used and Model Workflow, 3.2 Data Preprocessing, 3.3 Restricted Boltzmann Machines (RBMs), 3.4 Deep Belief Networks (DBNs), 3.5 Logistic Regression Classifier. We study RBMs ahead of DBNs because RBMs are used as a pre-training step for DBNs.

\subsection{Dataset Used and Model Workflow}

All the ECG signals are collected from MIT-BIH Arrhythmia dataset (Mark and Moody, 1998). This dataset is available publicly which has been widely used for performance evaluation of different ECGbased heartbeat classification algorithms. The dataset contains a total of 109446 records of patients. All records are classified into five categories as shown in Table 2.

A full automatic model for the detection process of arrhythmia disease classification with the help of ECG generated signals mainly requires four steps which are shown in Figure 1 and are:

1. ECG signal preprocessing, generating from the heartbeat signal of the patient.

2. Splitting of preprocessed signals into training and test set (8:2 split) and further into the training and testing sets of labels and data.

3. Normalization of training and testing data for better model performance.

4. Applying DBN for feature extraction on training data and labels.

5. Applying logistic regression classifier on enriched feature vector (output of DBN block) and testing for performance metrics on test data and labels.

Figure 2 shows a line graph of each class based on the data provided.

We further describe the types of heartbeats classified by N, S, V, F, and Q. 
Figure 1. Model workflow for feature enriched classification of ECG signals

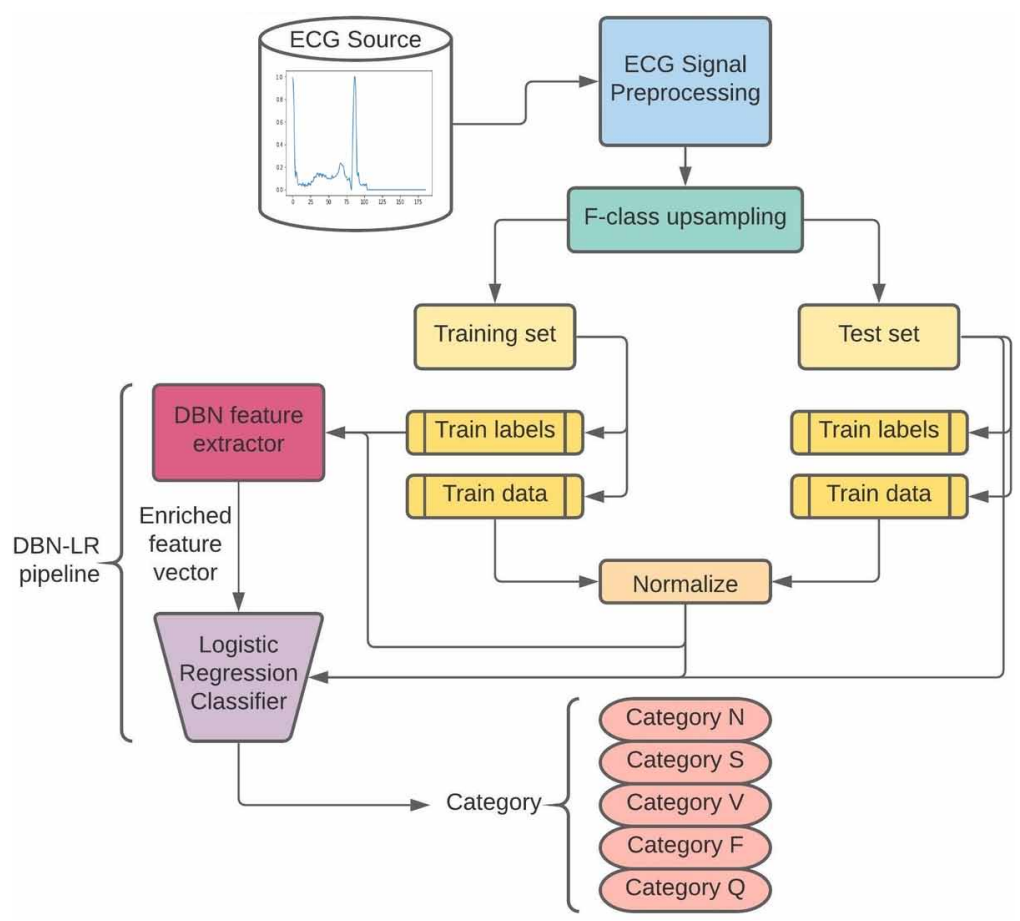

Table 2. Original composition of the number of class records

\begin{tabular}{|l|l|}
\hline \multicolumn{1}{|c|}{ Category } & \multicolumn{1}{c|}{ Number of records } \\
\hline $\mathrm{N}$ & 72471 \\
\hline $\mathrm{S}$ & 2223 \\
\hline $\mathrm{V}$ & 5788 \\
\hline $\mathrm{F}$ & 641 \\
\hline $\mathrm{Q}$ & 6431 \\
\hline
\end{tabular}

Figure 2. Line graphs (a), (b), (c), (d) and (e) for classes N, S, V, F, Q respectively

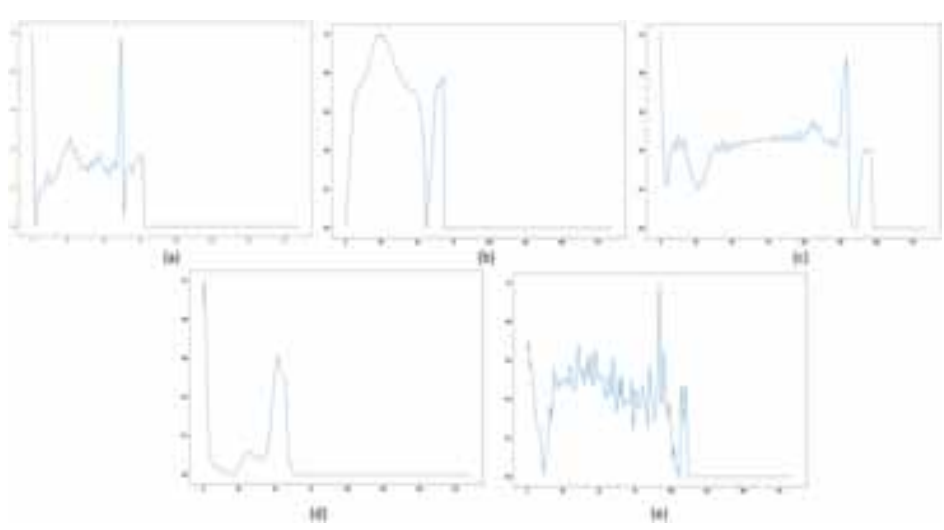


Figure 3. Ottesen et al. (2004). The four-chambered heart.

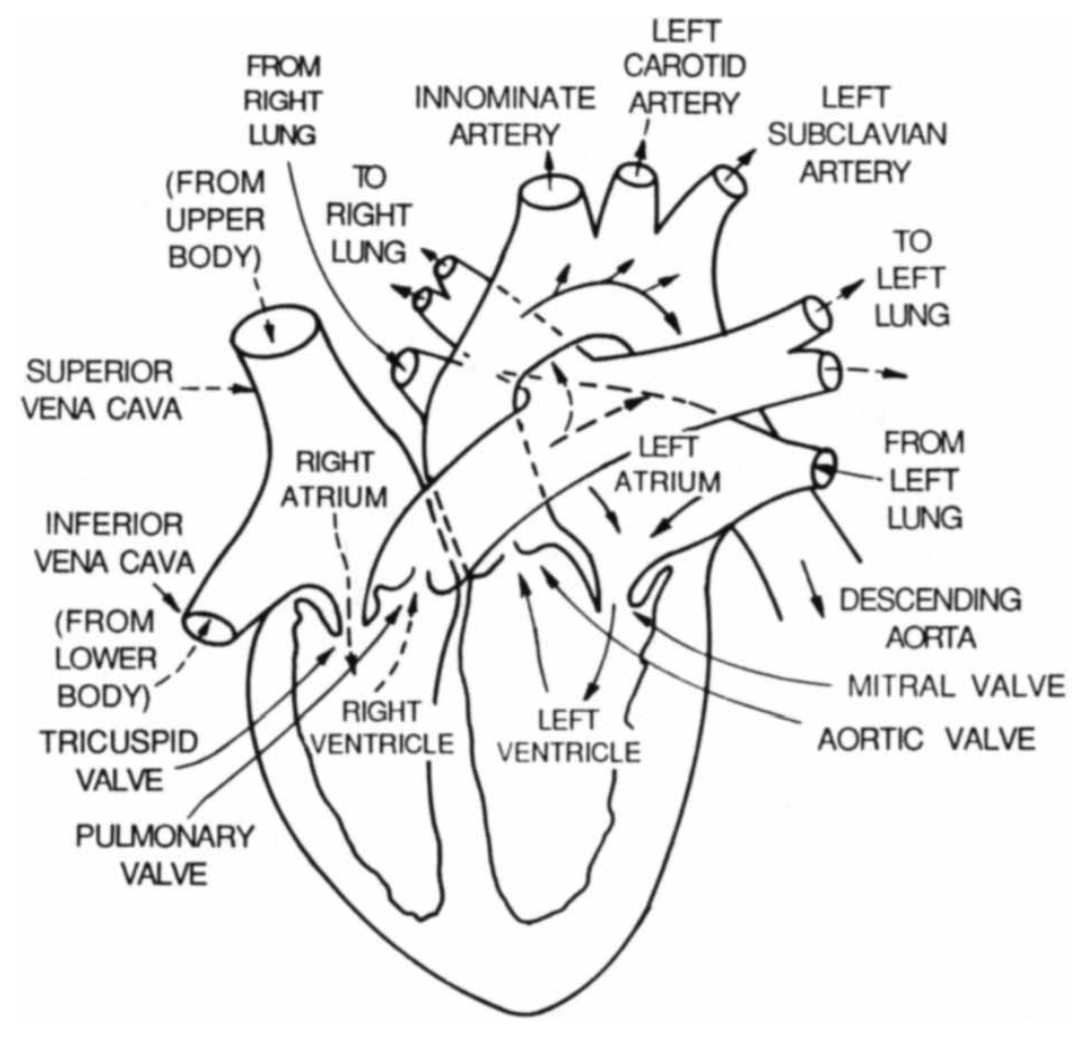

\subsubsection{N Categorization}

The $\mathrm{N}$ category beats are usually classified as normal heartbeats or left / right bundle branch block. The heart is composed of 4 chambers (the upper two are the right and left atria while the lower two are called ventricles) as shown in Figure 3. The electrical signal of the initialization of a heartbeat starts at the right atrium which travels to the left atrium and from there down to the ventricles. There are two bundle branches responsible for the conduction of the electrical signal to the ventricular section so those may beat. These bundles are termed the left and the right bundle. However, there may be a problem with the left or right branch of the conduction system which is termed as the left or right bundle branch block accordingly. In this problem, the signals do not normally travel downwards and are delayed due to the spread of the conduction signal from the right bundle branch via the cardiac muscle to gradually activate the left ventricle. When the left ventricle has a delayed contraction, there is a lack of coordination in the contraction of the cardiac muscles. This problem may be visible from the $\mathrm{N}$ categorization of the ECG signals.

\subsubsection{S Categorization}

Ectopic beats generally mean erratic heartbeat rhythms which may occur due to an earlier beat. Ectopic rhythm is also known as premature atrial contraction, extrasystole, and premature ventricular contraction. Usually, when this occurs, there is an early beat followed by a bolder beat of the heart. This occurs generally with normal, healthy people occasionally. Premature atrial contraction refers to the early beat originating from the right or left atria. This condition is very common among healthy children and is virtually harmless. Likewise, when the early beat arises from the ventricular region 
Figure 4. Addition of Gaussian noise with input ECG signals. The input signal (left) transforms to a new signal with added Gaussian noise (right).

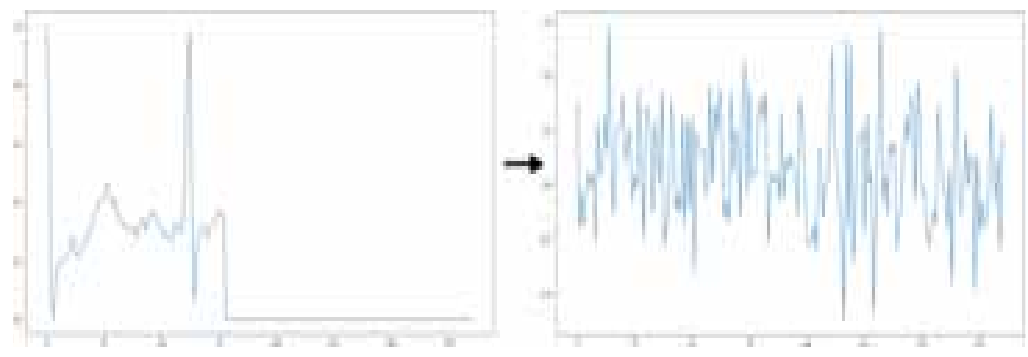

of the heart, is known as premature ventricular contraction. The risk of having premature ventricular contractions increases with age.

\subsubsection{Categorization}

Ventricular escape occurs when the electrical conduction system fails due to a heart block of all the bundles. This generates an impulse from a ventricular focus. These may even occur after long pauses in ventricular rhythm and is an impulsive action to avoid cardiac arrest. When the conduction system fails, there is an absence of heartbeats since the heart is unable to simulate the ventricles, which is when the ventricular escape is noticed.

\subsubsection{F Categorization}

The F class represents the fusion of ventricular ectopic and normal heartbeats.

\subsubsection{Q Categorization}

The heartbeats which are not classifiable according to the above conventions are placed into this category. The fusion of paced and normal beats along with only paced are also categorized into the Q class.

\subsection{Data Pre-Processing}

Usually, deep learning algorithms require large datasets to be trained upon for smooth functioning. We notice from Table 2 that there is a scenario of data imbalance. Class F only contains 641 instances as compared to class $\mathrm{N}$ with an overwhelming 72471 number of instances. We solve this by augmenting or resampling the data of class F and increasing it to 2000 instances in which new instances are made through random duplication. Likewise, 2000 instances of each class are taken to move forward with a balanced dataset.

Secondly, we apply a random Gaussian noise represented by the probability density function $P_{g}(z)$ given by:

$$
P_{g}(z)=\frac{1}{\sigma \sqrt{2 \pi}} \exp \left(-\frac{(z-\mu)^{2}}{2 \sigma^{2}}\right)
$$

In eqn. (1), $z$ denotes the grey level, with $\mu$ and $\sigma$ being the mean and standard deviation. We draw random samples from a Gaussian distribution centered at the origin and a standard deviation of 0.05 and add to the input signals to increase the variability of the data for generalization and better model performance. Figure 4 shows the effects of Gaussian noise on the input signal. 
Figure 5. Typical Boltzmann Machine where maroon circles represent theoretically visible nodes and blue ones represent hidden nodes

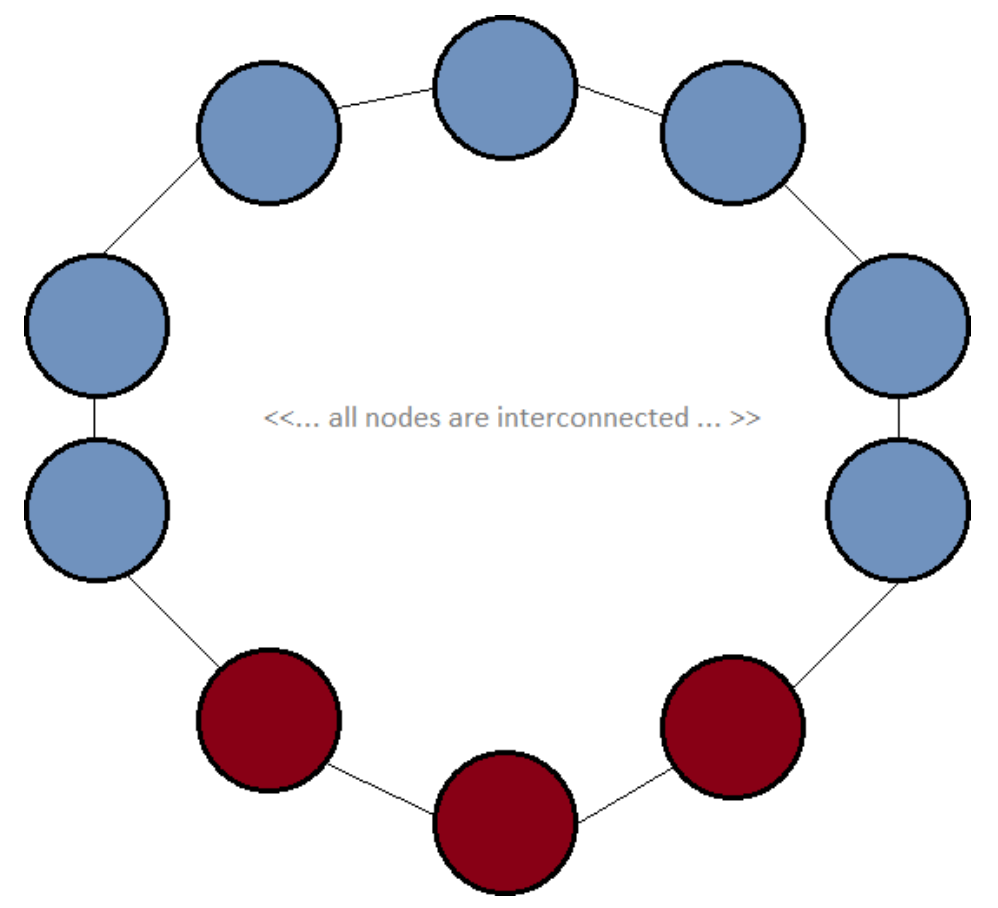

Thirdly, we normalize the test and train data as depicted by Figure 1 as:

$$
f_{\text {norm }}\left(x_{i}\right)=\frac{x_{i}-\min (x)}{\max (x)-\min (x)}
$$

Normalization of values is necessary for setting a common scale so that the machine learning algorithms can efficiently learn.

\subsection{Restricted Boltzmann Machines (RBMs)}

Restricted Boltzmann Machines are a derivative of the parent Boltzmann Machine (BM) model shown by Figure 5 .

BMs comprise many interlinked nodes having weighted connections. BMs represent the unsupervised categorization of deep learning models which generate different states of a given input system to model it. They are one of the prominent generative models in machine learning which create data that closely resembles the given input. After the input is provided to the input nodes, through a method named contrastive divergence, involving a process named Gibbs' sampling which is a technique of the Monte Carlo family, the hidden nodes feed the visible nodes in an iterative manner, and also the visible nodes feed the hidden ones, being multiplied by the weighted connections between them. The problem with this architecture was the large overhead on CPUs due to the many connections involving all the nodes being connected to all the other nodes. The RBM solves this problem.

To solve this, RBMs do away with the connections between the same types of nodes. In other words, no connections are made between visible and hidden nodes, and vice-versa. Doing so results 
Figure 6. Restricted Boltzmann Machine

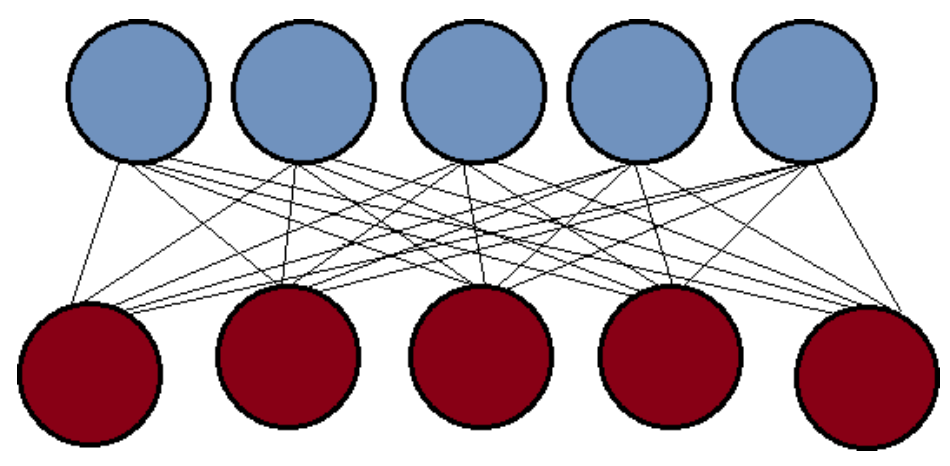

in a structure illustrated by Figure 6. BMs and RBMs generally are energy-based models (LeCun, 2006) which define a joint configuration $E(v, h)$ (Hopfield, 1982) which can be illustrated as:

$E(v, h)=-\sum_{i \in v i s i b l e} p_{i} v_{i}-\sum_{j \in \text { hidden }} q_{j} h_{j}-\sum_{i} \sum_{j} v_{i} w_{i, j} h_{j}$

The terms $h_{j}$ and $v_{i}$ refer to the hidden node $j$ and visible node $i$ respectively. Between these nodes, the weights and biases are given by $w_{i, j}$, and $q_{j}$ and $p_{i}$ respectively. The probability $p(v, h)$ is assigned as follows:

$$
p(v, h)=\frac{\exp (-E(v, h))}{\sum_{v, h} \exp (-E(v, h))}
$$

Through contrastive divergence, a gradient for learning is acquired as given by eqn. (5) using which the RBM determines the lowest energy state (which is also the most probable state of the system) through adjustment of weights:

$$
\frac{\delta \log p\left(v^{\text {initial }}\right)}{\delta w_{i, j}}=\left\langle v_{i}^{\text {initial }} h_{j}^{\text {initial }}\right\rangle-\left\langle v_{i}^{\text {final }} h_{j}^{\text {final }}\right\rangle
$$

\subsection{Deep Belief Networks (DBNs)}

DBNs may be thought of as RBMs stacked on top of each other. DBNs have a complex training procedure to eliminate problems such as the independence of the prior situated at the last hidden layer and another phenomenon called explaining away. Explaining away is a phenomenon observed in Bayesian networks where inference of hidden variables in hidden layers is intractable because their posterior over hidden variables is also intractable. In simpler terms, explaining away is observed when, one of the causes provides an explanation of the effect. This results in the reduction of the probability of occurrence of other causes. Let us take a logistic belief network that has randomly initialized binary units (Neal, 1992) to help generate data. All of unit $i$ is probabilities of activations 
are a sigmoidal function of the unit's prior neighbours $j$ and the weights among them $w_{i, j}$ (where the connections are as $i \rightarrow j$ ). For all neighbours of unit $i$, given by the prior $j$, there is a sigmoidal function. If $w_{i, j}$ denotes the weights amongst these units, then:

$$
P\left(s_{i}=1\right)=\frac{1}{1+\exp \left(-b_{i}-\sum_{j} s_{j} w_{i, j}\right)}
$$

In eqn. (6), $b_{i}$ is the bias of $i^{\text {th }}$ unit. In our experiments, we consider there to be only one hidden layer which can be termed as factorial. This means that all the hidden units that have conditional independence. However, due to the likelihood term propagating through from the input vector, the same is not true for the posterior. In (Hinton et al., 2006), authors proposed to that the problematic explaining away can be eliminated with the existence of a complimentary prior that has an opposite correlation to the likelihood term. A factorial posterior is obtained because of the product of prior and likelihood term.

Data generation in an infinite belief network is shown in Figure 7 which is similar to RBM's method of generation. The hidden nodes are connected to visible nodes and vice-versa. The RBM is can be seen as a reduction of the traditional BM to be sized down to a two-layer $(v, h)$ RBM having numerous contrastive divergence walks. This is known as the pre-training of the RBM before training of DBN. Authors in (Hinton et al., 2006) proposed a greedy learning algorithm for DBN that learns layer-wise as we describe below.

As described above, initially an RBM is trained to learn the data $x$. Let $R\left(c^{1} \mid c^{0}\right)$ be the posterior over $c^{1}$ where $c^{0}$ is the input data vector $x$. Now, we obtain an empirical distribution $\hat{d}^{1}$ over the layer $c^{1}$ when sampling $c^{0}$ from $\hat{d}$ (Bengio et al., 2007):

$$
\hat{d}^{1}\left(c^{1}\right)=\sum_{c^{0}} \hat{d}\left(c^{0}\right) R\left(c^{1} \mid c^{0}\right)
$$

Now, once the RBM is trained, it is placed atop the DBN at position $(n+1)$. Thus, $R\left(c^{n-1} \mid c^{n}\right)$ corresponds to $D\left(c^{n-1} \mid c^{n}\right)$ where $D($.$) represents the distribution of the posterior of the DBN.$ Thus, we can say that $R\left(c^{n-1} \mid c^{n}\right)$ is approximately equivalent to the posterior $D\left(c^{n-1} \mid c^{n}\right)$. Now we can eliminate the explaining away effect as the deepest hidden layer now has an independent prior. Thus, the elimination of explaining away is possible because of the independence of the prior at the last hidden layer. Using eqn. (7), it is possible to show:

$$
\hat{d}^{n}\left(c^{n}\right)=\sum_{c^{n-1}} \hat{d}^{n-1}\left(c^{n-1}\right) R\left(c^{n} \mid c^{n-1}\right)
$$

Using eqn. (8) the samples $c^{n-1}$ with distribution $\hat{d}^{n-1}$ are stochastically transformed to $c^{n}$ with distribution $\hat{d}^{n}$. Sampling from $c^{n}$ in an unbiased manner is now possible which is then fed downward stochastically by $R\left(c^{i} \mid c^{i-1}\right)$. Now we have an approximate posterior of $x$ clamped at $c^{0}$ by the lower hidden units. We can approximate posteriors $D\left(c^{i} \mid c^{0}\right)$ through mean-field approximation (as proposed in (Hinton et al., 2006)) by transformation of samples $c_{j}^{i-1}$ from level $i-1$ where 
Figure 7. The weights in the infinite logistic belief net are tied. When data flows from down to up, the sampling of inference from posterior distribution takes place and this is not related to generative modelling of the DBN. In the opposite sense, the generative model can be represented by the downward arrows. (Right): The bidirectional connections between two deep-most layers represent an RBM and the connections flowing from top to down are the generative model. The bottom to up connections infer a factorial representation from the layer beneath each layer. The bottom-up and top-down weights are initially tied in greedy learning.
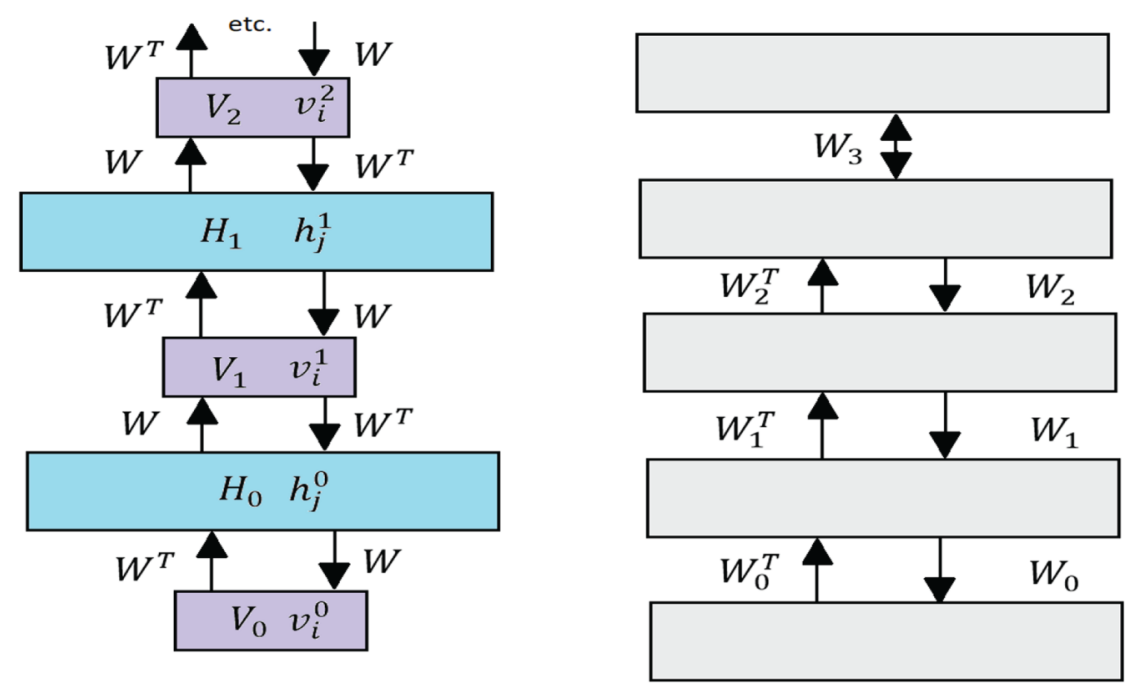

$j \in\left\{0,1, \ldots, m^{i}-1\right\}$ as $m^{i}$ represents the total number of units in the $i^{\text {th }}$ layer. Transformation of each sample via mean-field expected value $E_{j}^{i-1}$ can be done where:

$$
E^{i}=\sigma\left(b_{j}^{i}+W^{i} E^{i-1}\right)
$$

where:

$$
\sigma(x)=\frac{1}{1+e^{-x}}
$$

Eqn. (10) is called the sigmoid function used in logistic models (as we shall also see in Section. 3.5). Eqn. (10) is most commonly termed as the logistic or the sigmoid function. The weight matrix pertaining to the $i^{\text {th }}$ layer is given by $W^{i}$, while the bias of the unit $j$ situated at the $i^{\text {th }}$ layer is given by $b_{j}^{i}$. Authors in (Hinton et al., 2006) put forward the generic DBN equations as follows:

$$
\begin{aligned}
& D\left(c^{i} \mid c^{i+1}\right)=\prod_{j=1}^{m^{i}} D\left(c_{j}^{i} \mid c^{i+1}\right) \\
& D\left(c_{j}^{i}=1 \mid c^{i+1}\right)=\sigma\left(b_{j}^{i}+\sum_{k=1}^{m^{i+1}} W_{k j}^{i} c_{k}^{i+1}\right)
\end{aligned}
$$


Table 3. DBN configuration settings

\begin{tabular}{|l|l|l|l|l|}
\hline Hidden Layer Structure & Batch Size & Learning Rate (RBM) & \# Epochs & Activation Function \\
\hline$\{256,512\}$ & 10 & 0.06 & 10 & Sigmoid \\
\hline
\end{tabular}

\subsection{Logistic Regression Classifier}

The logistic regression classifier is a simple machine learning model which learns the relationships or the patterns represented between binary variables. Given a vector of $n$ independent variables by set $X=\left\{x_{1}, x_{2}, \ldots, x_{n}\right\}$, the target variable $Y$ (or, dependent variable) is calculated through eqn. (13) where $p=P(Y=1)$ :

$\log _{e}\left(\frac{p}{1-p}\right)=\beta_{0}+\beta_{1} x_{1}+\beta_{2} x_{2}+\ldots+\beta_{n} x_{n}$

Solving eqn. (13) further, we can obtain the logistic sigmoid function as seen earlier in eqn. (10):

$p=\frac{1}{1+e^{-\left(\beta_{0}+\beta_{1} x_{1}+\beta_{2} x_{2}+\ldots+\beta_{n} x_{n}\right)}}$

\section{EXPERIMENTATION AND RESULTS}

In this section, we present the results of training a logistic regression classifier on a feature vector selected by training the DBN on the ECG MIT-BIH dataset. All experiments performed were done using Anaconda Python 3.5 on a CPU having 8 GB RAM with Intel Core i5 $8^{\text {th }}$ generation $1.8 \mathrm{GHz}$ processor on Windows 10 with NVIDIA MX130 GPU. We specify the configuration settings for training the DBN in Table 3 . The hidden layer structure $\{256,512\}$ refers to having 256 hidden nodes in the first hidden layer and 512 hidden nodes in the second hidden layer. The RBM reconstruction errors encountered in each epoch are given in Figure 8. We train the algorithm for only 10 epochs since is clear from Figure 8 that the model converges at epoch 8 and the error is constant henceforth. Further training may have resulted in overfitting.

To compare our approach, we use another approach using 1D convolutional neural networks. Convolutional neural networks have been used for the detection of ECG by various researchers (Huang et al., 2019; Jun et al., 2018; Sharma et al., 2020). The configuration of this network is given in Table 4. The total number of parameters for the model was 118,341 with 117,957 trainable and 384 non-trainable parameters. As for training history, the model accuracy and loss have been plotted in Figure 9 and Figure 10 respectively.

To obtain a comparative result, we train the same dataset on the raw preprocessed data and feed it into the logistic classifier without selection of features by DBN and illustrate how much the results vary. Table 8 shows the performance difference of the three approaches based on five metrics accuracy, precision, recall, specificity, and F1-score. These metrics are defined as:

$$
\text { Accuracy }=\frac{(T P+T N)}{(T P+F P+F N+T N)}
$$


Figure 8. RBM reconstruction error encountered during pre-training the DBN

\section{RBM Reconstruction Error}

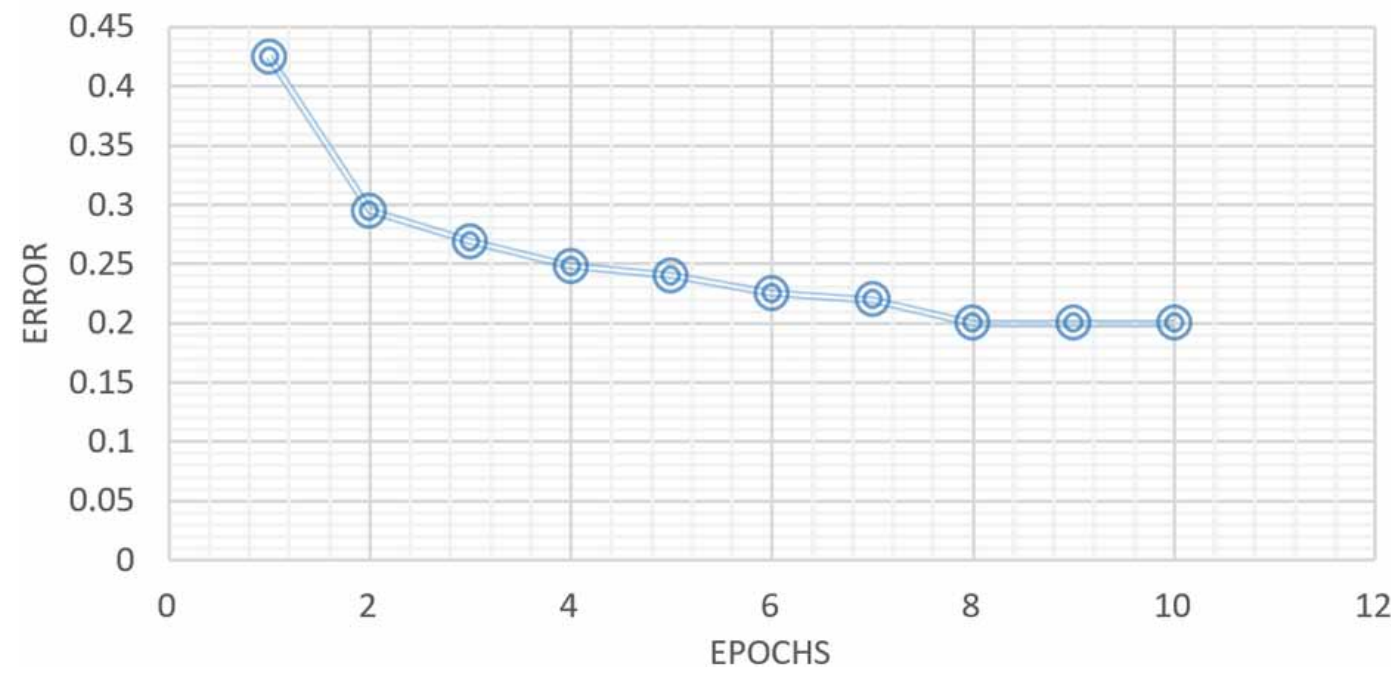

Table 4. 1D convolutional neural network configuration. The algorithm was trained using TensorFlow's Keras on Python 3.

\begin{tabular}{|l|l|l|l|l|}
\hline \multicolumn{1}{|c|}{ Layer (no. of filters) } & \multicolumn{1}{|c|}{ Size } & \multicolumn{1}{c|}{ Activation } & \multicolumn{1}{c|}{ Output shape } & \multicolumn{1}{c|}{ Parameters } \\
\hline Conv1D (64) & $6 \times 6$ & ReLU & (None, 181, 64) & 448 \\
\hline BatchNorm & - & - & (None, 181, 64) & 256 \\
\hline MaxPooling1D & $3 \times 3$ & - & (None, 91, 64) & 0 \\
\hline Conv1D (64) & $3 \times 3$ & ReLU & (None, 89, 64) & 12352 \\
\hline BatchNorm & - & - & (None, 89, 64) & 256 \\
\hline MaxPooling1D & $2 \times 2$ & - & (None, 45, 64) & 0 \\
\hline Conv1D (64) & $3 \times 3$ & ReLU & (None, 43, 64) & 12352 \\
\hline BatchNorm & - & - & (None, 43, 64) & 256 \\
\hline MaxPooling1D & $2 \times 2$ & - & (None, 22, 64) & 0 \\
\hline Flatten & - & - & (None, 1408) & 0 \\
\hline Dense & 64 & ReLU & (None, 64) & 90176 \\
\hline Dense & 32 & ReLU & (None, 32) & 2080 \\
\hline Dense_output & 5 & Softmax & (None, 5) & 165 \\
\hline
\end{tabular}


Figure 9. 1D convolution training history in terms of accuracy

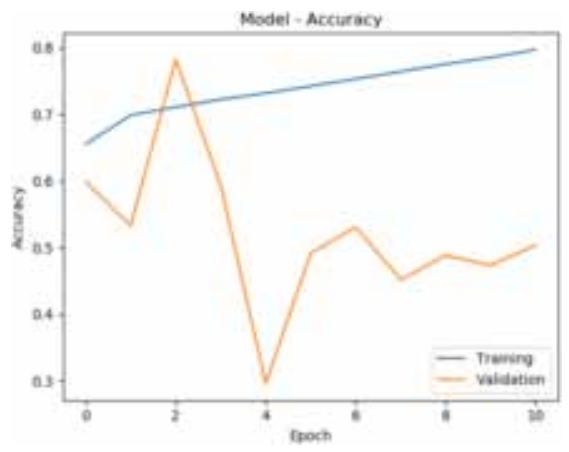

Precision $=\frac{T P}{(T P+F P)}$

Recall $=\frac{T P}{(T P+F N)}$

Specificity $=\frac{T N}{(T N+F P)}$

Figure 10. 1D convolution training history in terms of loss

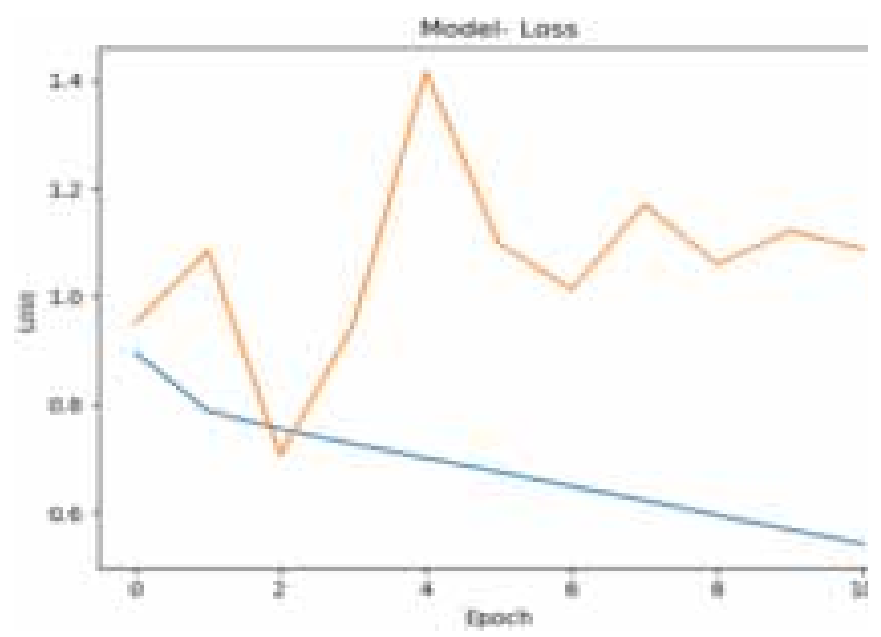


Table 5. Confusion matrix of DBN with extracted features

\begin{tabular}{|c|c|c|c|c|c|}
\hline & $\mathbf{N}$ & $\mathbf{S}$ & $\mathbf{V}$ & $\mathbf{F}$ & $\mathbf{Q}$ \\
\hline $\mathbf{N}$ & $\mathbf{1 4 0 6 6}$ & 67 & 7 & 17 & 11 \\
\hline $\mathbf{S}$ & 256 & $\mathbf{3 6 1}$ & 2 & 3 & 6 \\
\hline $\mathbf{V}$ & 65 & 8 & $\mathbf{1 1 3 7}$ & 11 & 5 \\
\hline $\mathbf{F}$ & 63 & 3 & 8 & $\mathbf{3 6 4}$ & 1 \\
\hline $\mathbf{Q}$ & 42 & 4 & 2 & 4 & $\mathbf{1 2 6 2}$ \\
\hline
\end{tabular}

$\mathrm{F} 1=2\left(\frac{\text { Precision } \times \text { Recall }}{\text { Precision }+ \text { Recall }}\right)=\frac{2 T P}{2 T P+F P+F N}$

In eqns. (15), (16), (17), (18), and (19), TP, TN, FP, and $F N$ refer to true positive, true negative, false positive, and false negative respectively. We further define the terms precision, recall, specificity, and F1 score in terms of a binary classification problem. Precision is defined as the ratio of the positive observations that the model correctly predicted to the total number of predicted positive observations. Whereas, recall, also referred to as sensitivity, is the ratio of the positive observations that the model correctly predicted to all the observations in the actual class of 'positive'. Specificity can be thought of as the probability that the prediction is negative given that the true observation is also negative. F1 score is just the weighted average of precision and recall. One important thing to note about using the F1 score on multi-class classification problems is that a higher F1 score does not always necessarily mean that it is a better classifier.

More specifically, the F1 score takes the weighted average of precision and recall. This means that it gives equal weight to both precision and recall. However, in classification problems (even binary), this is hardly the case. The importance of precision and recall varies from problem to problem. As an example, misclassifying a healthy person as a sick person does not have the same implication as misclassifying a sick person as healthy. In the same way, this importance of classification and misclassification of pairs of classes increases due to the increase in the number of possible pairs of classes in multi-class problems. Hence, F1 scores do not take into consideration if, say in our application, misclassifying $\mathrm{N}$ as $\mathrm{S}$ is more dangerous than misclassifying $\mathrm{S}$ as $\mathrm{N}$. Table 5 , Table 6 , and Table 6 are the confusion matrices generated by the DBN-LR model, LR model, and the 1D convolution model respectively. We calculate all our metrics from these results.

$\mathrm{P}, \mathrm{R}, \mathrm{S}$, and F1 refer to precision, recall, specificity, and F1 score respectively in Table 8. It can be clearly seen that feature extraction using DBN leads to superior results as compared to normal classification approaches without feature extraction. The classifier is better able to identify the classes

Table 6. Confusion matrix with no extraction of features

\begin{tabular}{|c|c|c|c|c|c|}
\hline & $\mathbf{N}$ & $\mathbf{S}$ & $\mathbf{V}$ & $\mathbf{F}$ & $\mathbf{Q}$ \\
\hline $\mathbf{N}$ & $\mathbf{1 2 6 1 3}$ & 113 & 74 & 36 & 92 \\
\hline $\mathbf{S}$ & 1052 & $\mathbf{3 0 1}$ & 14 & 15 & 37 \\
\hline $\mathbf{V}$ & 360 & 19 & $\mathbf{1 0 0 1}$ & 39 & 42 \\
\hline $\mathbf{F}$ & 281 & 4 & 63 & $\mathbf{2 9 6}$ & 23 \\
\hline $\mathbf{Q}$ & 187 & 6 & 5 & 13 & $\mathbf{1 0 9 0}$ \\
\hline
\end{tabular}


Table 7. Confusion matrix for 1D convolution approach

\begin{tabular}{|c|c|c|c|c|c|}
\hline & $\mathbf{N}$ & $\mathbf{S}$ & $\mathbf{V}$ & $\mathbf{F}$ & $\mathbf{Q}$ \\
\hline $\mathbf{N}$ & $\mathbf{1 7 9 8 5}$ & 26 & 26 & 39 & 42 \\
\hline $\mathbf{S}$ & 375 & $\mathbf{1 5 6}$ & 24 & 0 & 1 \\
\hline $\mathbf{V}$ & 736 & 20 & $\mathbf{5 4 5}$ & 4 & 143 \\
\hline $\mathbf{F}$ & 112 & 0 & 1 & $\mathbf{4 8}$ & 1 \\
\hline $\mathbf{Q}$ & 237 & 0 & 0 & 0 & $\mathbf{1 3 7 1}$ \\
\hline
\end{tabular}

of the ECG signals given a more specific set of data rather than the whole data of the signals. Our DBN is successfully able to do this by extracting important hidden features from the signals and feeding them into the logistic classifier. We compute the macro average for the metrics precision and recall achieved by our DBN-Logistic model to be 0.91 and 0.928 respectively. From Table 8 it is evident that the DBN-LR pipeline augments the detection accuracy by $10 \%$ and also outperforms the $1 \mathrm{D}$ convolution detection accuracy on various metrics including accuracy.

The bold values in Table 8 should be interpreted class-wise (i.e. row-wise). These values indicate the highest value in the row, and as a result of multiple highest values, all such instances have been made bold in the same row. It can be seen that although 1D convolution also compares with our approach in terms of precision on class $\mathrm{N}$, specificity on class $\mathrm{S}$, and specificity on class $\mathrm{F}$. However, most of the highest values reside in the DBN extracted features block and this is also supported by the highest accuracy of $96.7 \%$ achieved by our approach.

These results are promising as DBN can be used as an efficient feature extractor. These features can then be fed into prominent classifiers (in this study, we have only used logistic regression). Further, we compare our results with some of the results attained by other works in the literature as listed in Table 9. It is important to note that the final accuracy achieved is not the main aspect to be inspected, however, it is, in fact, the increase in the accuracy. This is because researchers may use DBN as a feature extractor instead of a logistic regression classifier as we have done. Convolutional neural networks are the gold standard when it comes to image classification, but we argue that DBN can also be considered as a competent algorithm for feature extraction in certain use-cases. Pipelines such as DBN-ANN (where ANN stands for artificial neural network), DBN-RF (random forest), and

Table 8. Comparison of feature extraction using DBN vs. no feature extraction vs. 1D convolution based on defined performance metrics

\begin{tabular}{|c|c|c|c|c|c|c|c|c|c|c|c|c|}
\hline \multirow[t]{2}{*}{ Class } & \multicolumn{4}{|c|}{ DBN extracted features } & \multicolumn{4}{|c|}{ No feature extraction } & \multicolumn{4}{|c|}{ 1D Convolution } \\
\hline & $\mathbf{P}$ & $\mathbf{R}$ & $\mathbf{S}$ & F1 & $\mathbf{P}$ & $\mathbf{R}$ & $\mathbf{S}$ & F1 & $\mathbf{P}$ & $\mathbf{R}$ & $\mathbf{S}$ & F1 \\
\hline $\mathrm{N}$ & 0.99 & 0.97 & 0.96 & 0.98 & 0.98 & 0.87 & 0.90 & 0.92 & 0.99 & 0.92 & 0.94 & 0.96 \\
\hline S & 0.57 & 0.81 & 0.98 & 0.67 & 0.21 & 0.68 & 0.93 & 0.32 & 0.28 & 0.77 & 0.98 & 0.41 \\
\hline $\mathrm{V}$ & 0.93 & 0.98 & 0.99 & 0.95 & 0.70 & 0.87 & 0.97 & 0.78 & 0.38 & 0.91 & 0.95 & 0.53 \\
\hline $\mathrm{F}$ & 0.83 & 0.91 & 0.99 & 0.87 & 0.44 & 0.81 & 0.97 & 0.57 & 0.30 & 0.53 & 0.99 & 0.38 \\
\hline Q & 0.96 & 0.98 & 0.99 & 0.97 & 0.84 & 0.85 & 0.98 & 0.84 & 0.85 & 0.88 & 0.98 & 0.87 \\
\hline Acc. & \multicolumn{4}{|c|}{0.967} & \multicolumn{4}{|c|}{0.863} & \multicolumn{4}{|l|}{0.91} \\
\hline
\end{tabular}

DBN-XGBoost could result in even better final accuracy. Moreover, the performance augment given 
Table 9. Comparison of our results with related work. Avg. in the column header indicates the average of all values obtained for different classes or types for the given metric

\begin{tabular}{|l|l|l|l|l|l|}
\hline \multicolumn{1}{|c|}{ Related Work } & Precision (avg.) & Recall (avg.) & Specificity (avg.) & F1-score (avg.) & Accuracy \\
\hline Acharya et al. (2017b) & 0.97 & 0.96 & 0.91 & N/A & 0.94 \\
\hline Sayadi et al. (2010) & 0.74 & 0.75 & N/A & N/A & 0.99 \\
\hline Prasad et al. (2013) & 0.98 & 0.98 & 0.98 & N/A & 0.98 \\
\hline Martis et al. (2011) & 0.98 & 0.98 & 0.98 & N/A & 0.97 \\
\hline Li et al. (2016) & 0.93 & 0.94 & - & N/A & 0.94 \\
\hline Our work & $\mathbf{0 . 9 1}$ & $\mathbf{0 . 9 2}$ & $\mathbf{0 . 9 8}$ & $\mathbf{0 . 8 8}$ & $\mathbf{0 . 9 6 7}$ \\
\hline
\end{tabular}

by DBN is not promised for every application - this means that DBN may not help with increasing accuracy when, say, it is applied for detection of breast cancer. More along these lines is provided in Section 5.1.

The metrics we achieve in this paper, in comparison to existing studies, may not be superior to them. However, as mentioned earlier, the important aspect of this research work is to examine whether DBN improves detection accuracy for a generic classifier when used as a feature extractor.

\section{FUTURE DIRECTIONS}

For future work the occurrence, sequential patterns, and persistence of five categories of ECG signals' heartbeats: N, S, V, F, and Q can be grouped in three prominent categories: Red, Yellow, and Green which represent danger, abnormal, and normal conditions of heart activity depending upon the electrical signals generated by ECG. We also insinuate the use of new techniques for capturing ECG signals (Ex: off-the-person approach), for the development of new datasets. The creation of such new datasets will be a challenging task not only because of the financial cost involved but also the dataset has to be incorporated into standards such as AAMI to reach the desired audience.

While working on this study we noticed that there are very few novel studies on feature extraction of ECG signals using Bayesian deep neural networks. These features are usually extracted in the time domain itself (Mazomenos et al., 2012) or its frequency domain transforms (Lin, 2008). More examples of such feature extractions are by the use of Karhunen-Loeve Transform, Hermitian Basis, Discrete Wavelet Transform, Short-Term Fourier Transform, etc. One possible future direction to take could be the transformation of ECG signals into one of the aforementioned transforms and then applying deep neural networks to extract features for even better performance of the predictive models. Further, we discuss certain strengths and limitations of our work. Moreover, as discussed earlier, researchers can use DBN as a preliminary feature extractor and apply different algorithms to create pipelines such as DBN-ANN, DBN-RF, DBN-XGBoost, etc. for better results.

\subsection{Strengths and Limitations}

In this study, we explored how DBNs can be used as a tool for amassing higher-order features for input to a classifier to improve performance metrics, as seen in Table 8. The main advantage of using DBN in this application is that we saw that the performance increase in terms of accuracy can be as high as $10 \%$. This augment in performance ability for any classifier has profound applications for other areas of medical research where the classification of diseases is crucial for patient prognoses (Jee et al., 2021; Yıldırım et al., 2018). This accuracy increase is also noticed when compared to 1D convolution. Moreover, the DBN, being a generative model, can generate its own samples based on the features it learned while training. If we sample the different representations from the DBN, we 
can understand what the model considered as important. This makes the model intelligible - in other words, it helps explain why the predictions were made the way they were. Intelligibility is critical in machine learning models when used for clinical applications, and hence, to understand the predictions, less powerful approaches with lesser accuracy may be chosen over more powerful, higher accuracyyielding approaches (like neural networks) which act essentially like a black-box. Hence, DBN acts as both, a powerful feature extractor and also as an intelligible model.

There are some limitations to this study. Hyperparameter tuning could have been done to find optimum parameters for the DBN, however, this would result in a long model forming process. Also, the methodology used here does not beat all performance metrics attained by other studies as seen in Table 9. This is because the DBN is a generative model and is better at generating data rather than having it being used as a discriminator. In this study, the DBN is used as a feature extractor and the way it does this is by generating features when given an input vector of ECG signals. The generated features for some classes, at times, maybe imprecise owing to the complexity of a quintuple classification problem. Training DBNs is also very computationally expensive as these run on Markov chains. Lastly, there do not exist any well-defined frameworks for the implementation of DBNs researchers are mostly left searching for viable solutions and code to their specific tasks and often are required to write their implementation from scratch. Hence, the flexibility of implementation of DBNs (finding more suitable versions of DBN implementations rather than those found on-line) is still an active area of application of unsupervised deep learning techniques.

\section{CONCLUSION}

In the proposed study, a deep learning approach is implemented for making an automatic categorizing heartbeat model with different ECG heartbeat signals which is an important step for the diagnosis of arrhythmia disease. DBN feature extraction pipelined with logistic regression classifier is used in the model. We compare this approach to (a) no feature extraction for logistic regression classifier, and (b) $1 \mathrm{D}$ convolutional neural network. The study shows that feature enrichment plays an important role in accurately classifying the data, as with feature extraction the accuracy improved from $86 \%$ to $96 \%$. Similar results were observed as the DBN-Logistic model gave superior results to 1D convolution (5\% increase in accuracy). The experiments were performed on the MIT-BIH dataset and the proposed method is found to be efficient and accurate based on the performance metrics of precision, recall, specificity, F1 score and accuracy. Based on the results achieved by the model, the same methods can be implemented into computer-aided design (CAD) ECG systems for getting quick, efficient, and reliable performance for diagnosis. Our model can be implemented in polyclinics to online and offline screen a large number of ECG recordings to reduce the waiting time of patients as well as reduce the workload on cardiologists and the cost of ECG signal processing. 


\section{REFERENCES}

Acharya, U. R., Fujita, H., Lih, O. S., Hagiwara, Y., Tan, J. H., \& Adam, M. (2017). Automated detection of arrhythmias using different intervals of tachycardia ECG segments with convolutional neural network. Information Sciences, 405, 81-90. doi:10.1016/j.ins.2017.04.012

Acharya, U. R., Oh, S. L., Hagiwara, Y., Tan, J. H., Adam, M., Gertych, A., \& Tan, R. S. (2017). A deep convolutional neural network model to classify heartbeats. Computers in Biology and Medicine, 89, 389-396. doi:10.1016/j.compbiomed.2017.08.022 PMID:28869899

Acharya, U. R., Oh, S. L., Hagiwara, Y., Tan, J. H., Adeli, H., \& Subha, D. P. (2018). Automated EEG-based screening of depression using deep convolutional neural network. Computer Methods and Programs in Biomedicine, 161, 103-113. doi:10.1016/j.cmpb.2018.04.012 PMID:29852953

Afonoso, V. X., \& Tompkins, W. J. (1995). Detecting ventricular fibrillation: Selecting the appropriate timefrequency analysis tool for the application. IEEE Engineering in Medicine and Biology Magazine, 14(2), 152-159. doi:10.1109/51.376752

Al Rahhal, M. M., Bazi, Y., AlHichri, H., Alajlan, N., Melgani, F., \& Yager, R. R. (2016). Deep learning approach for active classification of electrocardiogram signals. Information Sciences, 345, 340-354. doi:10.1016/j. ins.2016.01.082

Anand, A., Anand, H., Rautaray, S. S., Pandey, M., \& Gourisaria, M. K. (2020). Analysis and prediction of chronic heart diseases using machine learning classification models. International Journal of Advanced Trends in Computer Science and Engineering, 9(5), 8479-8487. doi:10.30534/ijatcse/2020/227952020

Association for the Advancement of Medical Instrumentation. (1998). Testing and reporting performance results of cardiac rhythm and ST segment measurement algorithms (ANSI/AAMI EC38). Author.

Bengio, Y., Lamblin, P., Popovici, D., \& Larochelle, H. (2007). Greedy layerwise training of deep networks, Proceedings of the 20th International Conference on Neural Information Processing Systems, 153-160.

Chandra, S., \& Gourisaria, M.K., GM, H., Rautaray, S.S., Pandey, M., \& Mohanty, S.N. (2021). Semantic Analysis of Sentiments through Web-Mined Twitter Corpus. CEUR Workshop Proceedings, 2786, 122-135.

Chen, S., Hua, W., Li, Z., Li, J., \& Gao, X. (2017). Heartbeat classification using projected and dynamic features of ECG signal. Biomedical Signal Processing and Control, 31, 165-173. doi:10.1016/j.bspc.2016.07.010

Das, S., Sharma, R., Gourisaria, M. K., Rautaray, S. S., \& Pandey, M. (2020). Heart Disease detection using Core Machine Learning and Deep Learning Techniques: A Comparative study. International Journal on Emerging Technologies, 11(3), 531-538.

De Chazal, P., O’Dwyer, M., \& Reilly, R. B. (2004). Automatic classification of heartbeats using ECG morphology and heartbeat interval features. IEEE Transactions on Biomedical Engineering, 51(7), 1196-1206. doi:10.1109/ TBME.2004.827359 PMID:15248536

Elhaj, F. A., Salim, N., Harris, A. R., Swee, T. T., \& Ahmed, T. (2016). Arrhythmia recognition and classification using combined linear and nonlinear features of ECG signals. Computer Methods and Programs in Biomedicine, 127, 52-63. doi:10.1016/j.cmpb.2015.12.024 PMID:27000289

GM, H., Gourisaria, M.K., Rautaray, S.S., Pandey, M. (2021b). Pneumonia detection using CNN through chest X-ray. Journal of Engineering Science and Technology, 16(1), 861-876.

GM, H., Gourisaria, M. K., Rautaray, S. S., \& Pandey, M. (2021a). UBMTR: Unsupervised Boltzmann MachineBased Time-Aware Recommendation System. Journal of King Saud University-Computer and Information Sciences.

GM, H., Gourisaria, M. K., Pandey, M., \& Rautaray, S. S. (2020). A comprehensive survey and analysis of generative models in machine learning. Computer Science Review, 38, 100285. doi:10.1016/j.cosrev.2020.100285

Guillén, S. G., Arredondo, M. T., Martin, G., \& Corral, J. M. F. (1990). Ventricular fibrillation detection by autocorrelation function peak analysis. Journal of Electrocardiology, 22, 253-262. doi:10.1016/S00220736(07)80136-1 PMID:2614311 
Hannun, A. Y., Rajpurkar, P., Haghpanahi, M., Tison, G. H., Bourn, C., Turakhia, M. P., \& Ng, A. Y. (2019). Cardiologist-level arrhythmia detection and classification in ambulatory electrocardiograms using a deep neural network. Nature Medicine, 25(1), 65-69. doi:10.1038/s41591-018-0268-3 PMID:30617320

Healthsquare. (2007). Heart disease. Conference on computational intelligence for modelling control and automation, 179-182.

Hinton, G., Deng, L., Yu, D., Dahl, G. E., Mohamed, A. R., Jaitly, N., Senior, A., Vanhoucke, V., Nguyen, P., Sainath, T. N., \& Kingsbury, B. (2012). Deep neural networks for acoustic modeling in speech recognition: The shared views of four research groups. IEEE Signal Processing Magazine, 29(6), 82-97. doi:10.1109/ MSP.2012.2205597

Hinton, G. E., Osindero, S., \& Teh, Y. (2006). A fast learning algorithm for deep belief nets. Neural Computation, 18(7), 1527-1554. doi:10.1162/neco.2006.18.7.1527 PMID:16764513

Hopfield, J. J. (1982). Neural networks and physical systems with emergent collective computational abilities. Proceedings of the National Academy of Sciences of the United States of America, 79(8), 2554-2558. doi:10.1073/ pnas.79.8.2554 PMID:6953413

Hu, Y. H., Palreddy, S., \& Tompkins, W. J. (1997). A patient-adaptable ECG beat classifier using a mixture of experts approach. IEEE Transactions on Biomedical Engineering, 44(9), 891-900. doi:10.1109/10.623058 PMID:9282481

Huang, J., Chen, B., Yao, B., \& He, W. (2019). ECG arrhythmia classification using STFT-based spectrogram and convolutional neural network. IEEE Access: Practical Innovations, Open Solutions, 7, 92871-92880. doi:10.1109/ACCESS.2019.2928017

Inan, O. T., Giovangrandi, L., \& Kovacs, G. T. A. (2006). Robust neural-network-based classification of premature ventricular contractions using wavelet transform and timing interval features. IEEE Transactions on Biomedical Engineering, 53(12), 2507-2515. doi:10.1109/TBME.2006.880879 PMID:17153208

Ince, T., Kiranyaz, S., \& Gabbouj, M. (2009). A generic and robust system for automated patient-specific classification of ECG signals. IEEE Transactions on Biomedical Engineering, 56(5), 1415-1426. doi:10.1109/ TBME.2009.2013934 PMID:19203885

Jadhav, S. M., Nalbalwar, S. L., \& Ghatol, A. A. (2010). Arrhythmia disease classification using artificial neural network model. 2010 IEEE International Conference on Computational Intelligence and Computing Research, 1-4. doi:10.1109/ICCIC.2010.5705854

Jee, G., Harshvardhan, G. M., \& Gourisaria, M. K. (2021, February 17). Juxtaposing inference capabilities of deep neural models over posteroanterior chest radiographs facilitating COVID-19 detection. Journal of Interdisciplinary Mathematics, 24(2), 299-325. Advance online publication. doi:10.1080/09720502.2020.1838061

Jiang, W., \& Kong, S. G. (2007). Block-based neural networks for personalized ECG signal classification. IEEE Transactions on Neural Networks, 18(6), 1750-1761. doi:10.1109/TNN.2007.900239 PMID:18051190

Jun, T. J., Nguyen, H. M., Kang, D., Kim, D., Kim, D., \& Kim, Y. H. (2018). ECG arrhythmia classification using a 2-D convolutional neural network. arXiv preprint arXiv:1804.06812.

Khadra, L., Al-Fahoum, A. S., \& Al-Nashash, H. (1997). Detection of life-threatening cardiac arrhythmias using the wavelet transformation. Medical \& Biological Engineering \& Computing, 35(6), 626-632. doi:10.1007/ BF02510970 PMID:9538538

Kiranyaz, S., Ince, T., \& Gabbouj, M. (2016). Real-time patient-specific ECG classification by 1-D convolutional neural networks. IEEE Transactions on Biomedical Engineering, 63(3), 664-675. doi:10.1109/ TBME.2015.2468589 PMID:26285054

Krizhevsky, A., Sutskever, I., \& Hinton, G. E. (2012). ImageNet classification with deep convolutional neural networks. Proceedings of Advances in Neural Information Processing Systems, 1097-1105.

Lagerholm, M., Peterson, C., Braccini, G., Edenbrandt, L., \& Sornmo, L. (2000). Clustering ECG complexes using hermite functions and self-organizing maps. IEEE Transactions on Biomedical Engineering, 47(7), 838-848. doi:10.1109/10.846677 PMID:10916254

LeCun, Y. (2006). A Tutorial on Energy-Based Learning. http://yann.lecun.com/exdb/publis/pdf/lecun06.pdf 
LeCun, Y., Boser, B. E., Denker, J. S., Henderson, D., Howard, R. E., Hubbard, W. E., \& Jackel, L. D. (1990). Handwritten digit recognition with a back-propagation network. Advances in Neural Information Processing Systems, 396-404.

Li, D., Zhang, J., Zhang, Q., \& Wei, X. (2017). Classification of ECG signals based on 1D convolution neural network. 2017 IEEE 19th International Conference on e-Health Networking. Applications and Services (Healthcom), 1-6.

Li, H., Yuan, D., Ma, X., Cui, D., \& Cao, Y. (2017). Genetic algorithm for the optimization of features and neural networks in ECG signals classification. Scientific Reports, 7(41011). doi:10.1038/srep41011 PMID:28139677

Li, P., Wang, Y., He, J., Wang, L., Tian, Y., Zhou, T.-S., Li, T., \& Li, J.-S. (2017). High-performance personalized heartbeat classification model for longterm ECG signal. IEEE Transactions on Biomedical Engineering, 64(1), 78-86. doi:10.1109/10.650355 PMID:27046844

Li, T. Y., \& Min, Z. (2016). ECG Classification Using Wavelet Packet Entropy and Random Forests. Entropy (Basel, Switzerland), 18(8), 1-16. doi:10.3390/e18080285

Lin, C. H. (2008). Frequency-domain features for ECG beat discrimination using grey relational analysis-based classifier. Computers \& Mathematics with Applications (Oxford, England), 55(4), 680-690. doi:10.1016/j. camwa.2007.04.035

Luz, E. J. da S., Schwartz, W. R., Cámara-Chávez, G., \& Menotti, D. (2016). ECG-based heartbeat classification for arrhythmia detection: A survey. Computer Methods and Programs in Biomedicine, 127, 144-164. doi:10.1016/j. cmpb.2015.12.008 PMID:26775139

Mar, T., Zaunseder, S., Martínez, J. P., Llamedo, M., \& Poll, R. (2011). Optimization of ECG classification by means of feature selection. IEEE Transactions on Biomedical Engineering, 58(8), 2168-2177. doi:10.1109/ TBME.2011.2113395 PMID:21317067

Mark, R., \& Moody, G. (1998). MIT-BIH Arrhythmia Database Directory. MIT Press.

Martis, R. J., Acharya, U. R., \& Min, L. C. (2013). ECG beat classification using PCA, LDA, ICA and Discrete Wavelet Transform. Biomedical Signal Processing and Control, 8(5), 437-444. doi:10.1016/j.bspc.2013.01.005

Martis, R. J., Acharya, U. R., Ray, A. K., \& Chakraborty, C. (2011). Application of Higher Order Cumulants to ECG Signals for the Cardiac Health Diagnosis. 2011 Annual International Conference of the IEEE Engineering in Medicine and Biology Society, 1697-1700. doi:10.1109/IEMBS.2011.6090487

Mazomenos, E. B., Chen, T., Acharyya, A., Bhattacharya, A., Rosengarten, J., \& Maharatna, K. (2012). A time-domain morphology and gradient based algorithm for ECG feature extraction. 2012 IEEE International Conference on Industrial Technology, 117-122. doi:10.1109/ICIT.2012.6209924

Mozaffarian, D., Benjamin, E. J., Go, A. S., Arnett, D. K., Blaha, M. J., Cushman, M., \& Turner, M. B. et al. (2015). Heart Disease and Stroke Statistics—2016 Update. Circulation, 133(4), 38-360. PMID:26673558

National Center for Health Statistics. (2005). With Chartbook on Trends in the Health of Americans. https:// www.ncbi.nlm.nih.gov/books/NBK20990/

National Centre for Health Statistics. (2003). Deaths: Leading causes for 2003. Author.

Nayak, S., Gourisaria, M. K., Pandey, M., \& Rautaray, S. S. (2019). Heart Disease Prediction Using Frequent Item Set Mining and Classification Technique. International Journal of Information Engineering \& Electronic Business, 11(6), 9-15. doi:10.5815/ijieeb.2019.06.02

Neal, R. M. (1992). Connectionist learning of belief networks. Artificial Intelligence, 56(1), 71-113. doi:10.1016/0004-3702(92)90065-6

Ottesen, J. T., Olufsen, M. S., \& Larsen, J. K. (2004). Applied mathematical models in human physiology. Society for Industrial and Applied Mathematics. doi:10.1137/1.9780898718287

Owis, M. I., Abou-Zied, A. H., Youssef, A. B., \& Kadah, Y. M. (2002). Study of features based on nonlinear dynamical modeling in ECG arrhythmia detection and classification. IEEE Transactions on Biomedical Engineering, 49(7), 733-736. doi:10.1109/TBME.2002.1010858 PMID:12083309 
Pan, S.-T., Hong, T.-P., \& Chen, H.-C. (2012). ECG signal analysis by using hidden Markov model. Proceedings of International Conference on Fuzzy Theory Applications, 288-293. doi:10.1109/iFUZZY.2012.6409718

Prasad, H., Martis, R. J., Acharya, U. R., Min, L. C., \& Suri, J. S. (2013). Application of higher order spectra for accurate delineation of atrial arrhythmia. 2013 35th Annual International Conference of the IEEE Engineering in Medicine and Biology Society (EMBC), 57-60. doi:10.1109/EMBC.2013.6609436

Rajpurkar, P., Hannun, A. Y., Haghpanahi, M., Bourn, C., \& Ng, A. Y. (2017). Cardiologist-level arrhythmia detection with convolutional neural networks. arXiv preprint arXiv:1707.01836.

Sahu, A. GM, H., \& Gourisaria, M. K. (2020). A Dual Approach for Credit Card Fraud Detection using Neural Network and Data Mining Techniques. 2020 IEEE 17th India Council International Conference (INDICON). https://www.doi.org/10.1109/INDICON49873.2020.9342462

Sannino, G., \& Pietro, G. D. (2018). A deep learning approach for ECG-based heartbeat classification for arrhythmia detection. Future Generation Computer Systems, 86, 455-466. doi:10.1016/j.future.2018.03.057

Sayadi, O., Shamsollahi, M. B., \& Clifford, G. D. (2010). Robust Detection of Premature Ventricular Contractions Using a Wave-Based Bayesian Framework. IEEE Transactions on Biomedical Engineering, 57(2), 353-362. doi:10.1109/TBME.2009.2031243 PMID:19758851

Sharma, R., Gourisaria, M. K., Rautray, S. S., Pandey, M., \& Patra, S. S. (2020). ECG Classification using Deep Convolutional Neural Networks and Data Analysis. International Journal of Advanced Trends in Computer Science and Engineering, 9(4), 5788-5795. doi:10.30534/ijatcse/2020/236942020

Sidney, S., Rosamond, W. D., Howard, V. J., \& Luepker, R. V. (2013). The "heart disease and stroke statistics-2013 update" and the need for a national cardiovascular surveillance system. Circulation, 127(1), 21-23. doi:10.1161/CIRCULATIONAHA.112.155911 PMID:23239838

Smith, S. C. Jr, Clark, L. T., Cooper, R. S., Daniels, S. R., Kumanyika, S. K., Ofili, E., Quinones, M. A., Sanchez, E. J., Saunders, E., \& Tiukinhoy, S. D. (2005). Discovering the full spectrum of cardiovascular disease: Minority Health Summit 2003: report of the Obesity, Metabolic Syndrome, and Hypertension Writing Group. Circulation, 111(10), 134-139. doi:10.1161/01.CIR.0000157743.54710.04 PMID:15769755

Spach, M.S, \& Kootsey, J. M. (1983). The nature of electrical propagation in cardiac muscle. American Journal of Physiology - Heart and Circulatory Physiology, 244(H), 3-22.

Teijeiro, T., Félix, P., Presedo, J., \& Castro, D. (2018). Heartbeat classification using abstract features from the abductive interpretation of the ECG. IEEE Journal of Biomedical and Health Informatics, 22(2), 409-420. doi:10.1109/JBHI.2016.2631247 PMID:27893401

Thakor, N. V., \& Zhu, Y. S. (1991). Applications of adaptive filtering to ECG analysis: Noise cancellation and arrhythmia detection. IEEE Transactions on Biomedical Engineering, 38(8), 785-794. doi:10.1109/10.83591 PMID:1937512

Thaler, M. S. (1999). The Only EKG Book You'll Ever Need (3rd ed.). Lippincott Williams \& Wilkins.

World Health Organization. (2014). Global status report on noncommunicable diseases 2014 (No. WHO/NMH/ NVI/15.1). World Health Organization.

Ye, C., Kumar, B. V. K. V., \& Coimbra, M. T. (2012). Heartbeat classification using morphological and dynamic features of ECG signals. IEEE Transactions on Biomedical Engineering, 59(10), 2930-2941. doi:10.1109/ TBME.2012.2213253 PMID:22907960

Yıldırım, Ö., Pławiak, P., Tan, R. S., \& Acharya, U. R. (2018). Arrhythmia detection using deep convolutional neural network with long duration ECG signals. Computers in Biology and Medicine, 102, 411-420. doi:10.1016/j. compbiomed.2018.09.009 PMID:30245122 
Mahendra Kumar Gourisaria is presently working as an Assistant Professor in the School of Computer Engineering at KIIT University, Bhubaneswar, Odisha. He has received his Master's degree in Computer Application from Indira Gandhi National Open University, New Delhi, and M. Tech in Computer Science and Engineering from Biju Patnaik University of Technology - Rourkela. He is pursuing his Ph.D. from KIIT Deemed to be University. He has experience of more than 18 years in academia and 7 years in research. He has published more than 35 research papers in different international journals, conferences, and book chapters of repute. He has also served as the organizing committee members of various conferences and workshops. His area of research includes Cloud Computing, Data Mining, Soft Computing, and Internet and Web Technology. He is a member of IAENG, UACEE, and a life member of ISTE, CSI, and ISCA.

Harshvardhan G. M. is currently pursuing B. Tech degree in KIIT, Deemed to be University, Odisha, India. He is a published author with his survey paper on generative models in Computer Science Review journal with many other works in their final stages or conditionally accepted for publication. His research interests include unsupervised and self-supervised deep learning methods, clinical applications of machine learning for medicine, generative models in machine learning, recommender systems for content delivery, energy-efficient task scheduling in cloud computing, topic modelling in natural language processing using latent Dirichlet allocation and internet of drones. Mr. GM has won national and state-level awards for literary abilities in vocabulary and essay competitions.

Rakshit Agrawal is currently pursuing B.Tech in KIIT, Deemed to be University, and is currently in 1st year. He has won gold medal in robotics and various other state-level robotics competitions. His research interest includes machine learning, neural networks, deep learning and cloud computing.

Sudhansu Shekhat Patra is currently an Associate Professor in the School of Computer Application, KIIT University, Bhubaneswar, India. He received his Master degree in Computer Application from Motilal Nehru National Institute of Technology, Allahabad, India, M.Tech (Computer Science \& Engg) from Utkal University, Bhubaneswar, India and Ph.D. in Computer Science from KIIT University, Bhubaneswar, India. His research interests include grid computing, Cloud Computing, Algorithms. He is a life member of Indian Society for Technical Education.

Siddharth Swarup Rautary is presently working as an Associate Professor at School of Computer Engineering, Kalinga Institute of Industrial Technology, Deemed to be University, Bhubaneswar Odisha, India. He has teaching and research experience of more than 9 years. He did his doctoral degree from Indian Institute of Information Technology, Allahabad, U.P., India His research interest includes Big Data Analytics, Image Processing, Intelligent Systems, Human Computer Interaction and similar innovative areas. His research contribution includes 05 coedited proceedings/books which include ASIC Springer series, more than 60 research publications in reputed conferences, book chapters and journals indexed in Scopus/SCI/ESCI and with a citation index of 1800 as on date. As an organizing chair he has organized 05 International conferences (ICCAN2017, ICCAN 2019, 16th ICDCIT 2020, FICTA 2016, FICTA 2017) and has been part of different core committees of other conferences and workshops. He has delivered invited talks in different workshops and conferences.

Manjusha Pandey is presently working as an Associate Professor in the School of Computer Engineering, Kalinga Institute of Industrial Technology, and Bhubaneswar. She has pursued her PhD from Indian Institute of Information Technology, Allahabad. She has more than 95 research publications to her credit in journals and conferences of repute. Her research interest areas include Wireless Sensor Network, Security and Privacy in Wireless Sensor Network, Data Analytics, Human Computer Interaction and Computer Networks. 